\section{Simultaneous Pneumatosis Cystoides Intestinalis and Pneumomediastinum in a Patient with Systemic Sclerosis}

\section{To the Editor:}

Interstitial lung disease (ILD) is a common pulmonary manifestation in patients with systemic sclerosis (SSc). Pneumomediastinum occasionally develops in patients with connective tissue diseases (CTD) and ILD, but is rare in $\mathrm{SSc}^{1,2,3}$. Although gastrointestinal (GI) involvement is common in patients with $\mathrm{SSc}$, pneumatosis cystoides intestinalis (PCI), which is characterized by the presence of gas-filled cysts in the intestinal wall, is a rare complication ${ }^{4}$. We encountered a patient with SSc who showed simultaneous occurrence of pneumomediastinum and PCI.

A 61-year-old Japanese woman had been diagnosed with SSc 6 years earlier based on the findings of proximal scleroderma, positive antinuclear antibody (1:640 with nucleolar and homogeneous patterns), positive antiScl-70 antibody (1:16), and ILD. Fingertip ulceration had developed 4 years before. Prednisolone ( $20 \mathrm{mg} /$ day) had been started 2 years before for her skin sclerosis. She had gradually lost her appetite and her weight had declined by $15 \mathrm{~kg}$ over the previous 6 months. She was admitted to our division because of poor appetite and abdominal distension. On admission, she was being treated with $10 \mathrm{mg} /$ day prednisolone. Physical examination revealed that her abdomen was distended and tympanic. Bilateral fine crackles were heard in the lung bases, but she had no respiratory symptoms. Plain abdominal radiographs showed marked dilation of the small bowel with prominent Kerckring's folds and linear lucencies along the bowel wall. Computed tomography of the thorax and abdomen revealed multiple linear and cystic accumulations of gas in the walls of the small bowel, as well as ascites (Figure 1). There was also a small amount of free air around the dilated stomach and under the diaphragm, which extended into the retroperitoneal space. Further, there was free air around the trachea and in the subcutaneous tissues of the chest wall (Figure 2). These findings confirmed the simultaneous presence of PCI and pneumomediastinum. Her radiological abnormalities improved after a few weeks of conservative therapy, consisting of bowel rest and high-flow oxygen $(5 \mathrm{l} / \mathrm{min})$ by a face mask.
Pneumomediastinum has been associated with a variety of underlying lung diseases including asthma, bronchiectasis, chronic obstructive pulmonary disease, and ILD ${ }^{5}$. As well as such diseases, numerous precipitating factors exist, such as vomiting, coughing, asthma exacerbation, and exercise. Among patients with CTD, dermatomyositis (DM) or amyopathic DM is most frequently complicated by pneumomediastinum ${ }^{6}$. However, it is rare in patients with SSc, with only 3 such cases reported in the English-language literature ${ }^{1,2,3}$. All 3 patients had ILD as an underlying disease. As precipitating factors, 1 patient developed pneumomediastinum after undergoing lung function tests, but the others had no obvious cause. Our patient had ILD as an underlying disease, but she had no precipitating factors.

PCI is associated with a wide variety of medical conditions. In patients with SSc, intestinal hypomotility leads to bacterial overgrowth and bowel distension, which could elevate the intraluminal pressure and force gas into the walls of the intestine ${ }^{4}$. On the other hand, pulmonary disease is one of the causes of PCI. Severe coughing can trigger alveolar rupture, which could result in introduction of air along vascular channels in the mediastinum, tracking downward to the retroperitoneum and then to the mesentery of the bowel. This could explain the simultaneous occurrence of PCI and pneumomediastinum, but some authors question the theory ${ }^{7}$. Although our patient had ILD, she had neither precipitating factors nor chest symptoms such as pain or discomfort. Thus, descending air that originated from the lungs is less likely in our case.

Pneumomediastinum is a rare complication of GI endoscopy, and it usually indicates perforation leading into the peritoneal cavity or the retroperitoneal space that results from trauma to the GI mucosa ${ }^{8}$. Air enters the bowel wall by the mucosal wound and ascends through the retroperitoneum, tracking upward to reach the mediastinum. This can occur without any overt evidence of perforation ${ }^{8}$. This ascending route from the intestine is more reasonable in our patient, since her chief complaint was abdominal distension, and abdominal radiographs showed marked small bowel dilation (similar to that after endoscopic insufflation).

Both PCI and pneumomediastinum are rare in patients with SSc. It is highly unlikely that 2 rare conditions would occur simultaneously. Rather,

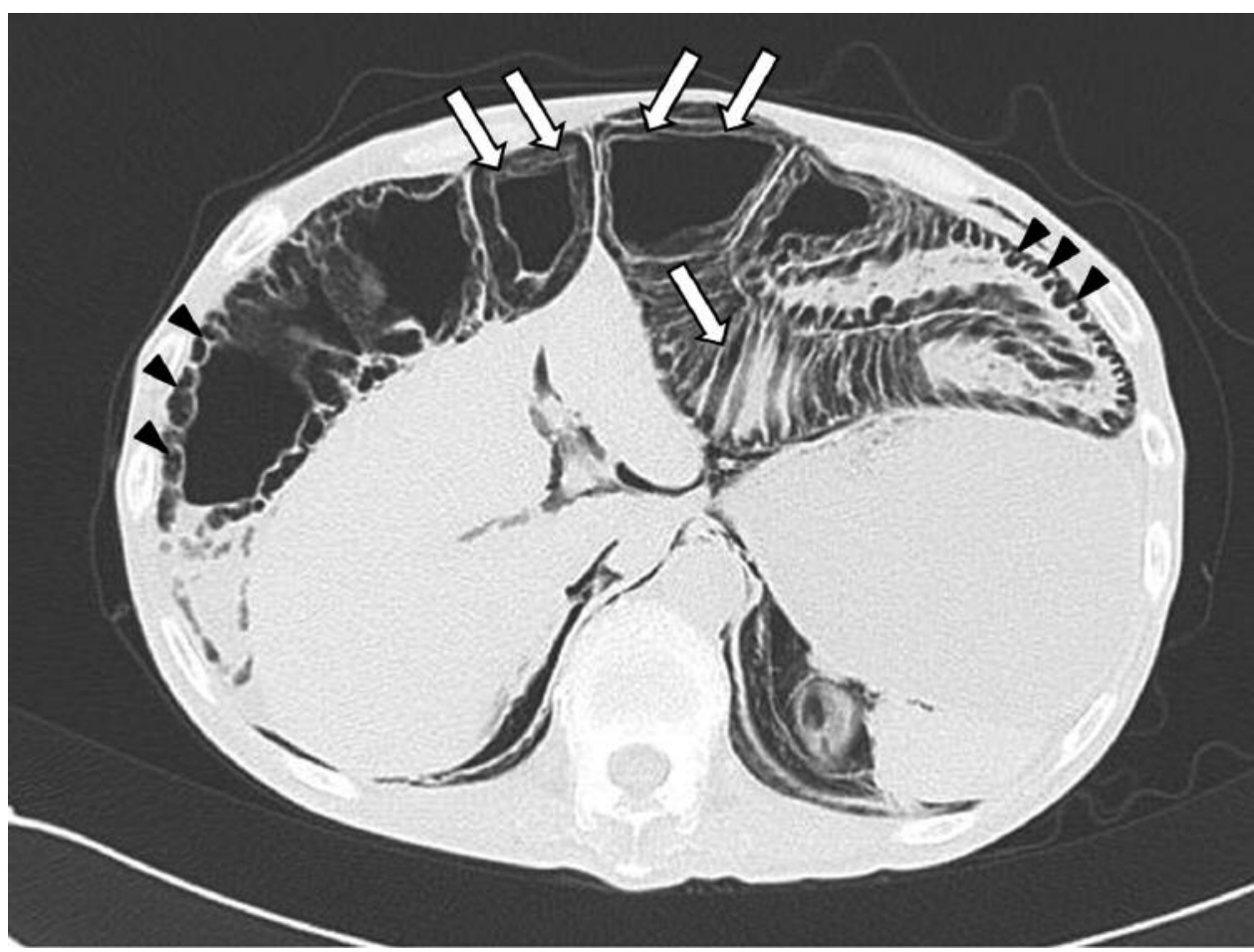

Figure 1. Cross-sectional computed tomography scan of the abdomen shows both cystic (arrowheads) and linear (arrows) accumulations of gas in the intestinal wall. 


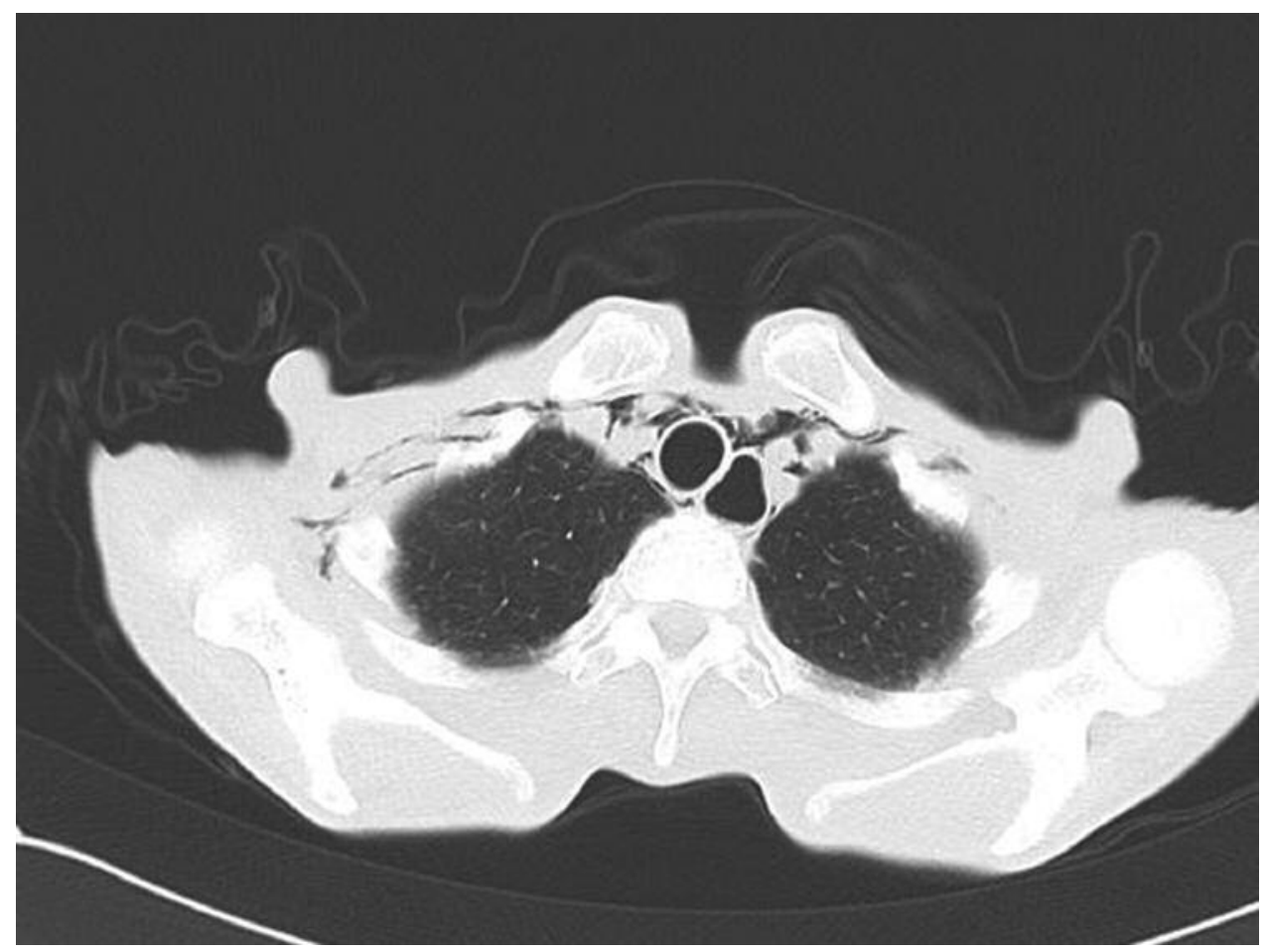

Figure 2. Computed tomography scan of the chest. There is free air around the trachea, which extends into the subcutaneous tissues of the anterior chest wall. The esophagus is also dilated.

it appears that retroperitoneal and peritoneal free air derived from PCI escaped upward along the para-aortic tissues and through the esophageal hiatus into the mediastinum, finally leading to pneumomediastinum and the subcutaneous emphysema in our patient. Unlike the previous cases in patients with SSc, this is not "spontaneous" pneumomediastinum. A similar case was reported in a patient with DM with simultaneous pneumomediastinum and $\mathrm{PCI}^{9}$. Corticosteroid therapy and/or rapid weight loss might be associated with loosening and weakening of the mediastinal connective tissues in such patients.

\section{KYOKO HONNE, MD; AKIHITO MARUYAMA, MD; SACHIKO} ONISHI, MD; TAKAO NAGASHIMA, MD, PhD; SEIJI MINOTA, MD, PhD, Professor of Medicine, Division of Rheumatology and Clinical Immunology, Department of Medicine, Jichi Medical University, Yakushiji 3311-1, Shimotsuke, Tochigi, 329-0498, Japan. Address correspondence to Dr. Nagashima; E-mail: naga4ma@jichi.ac.jp

\section{REFERENCES}

1. Mohammad A, Boon Low T, O’Dwyer D, McElvaney G, Kearns G. Spontaneous pneumo-mediastinum in systemic sclerosis: a case report. Rheumatology 2007;46:1376-7.

2. Jun JB, Song SY. The development of pneumomediastinum after pulmonary function testing in a patient with systemic sclerosis. Rheumatol Int 2007;27:1097-8.
3. Teixeira Moreira Almeida Mdo S, Dias LT, Fernandes SJ, Almeida JV. Spontaneous pneumomediastinum and subcutaneous emphysema in systemic sclerosis. Rheumatol Int 2007;27:675-7.

4. Sequeira W. Pneumatosis cystoides intestinalis in systemic sclerosis and other diseases. Semin Arthritis Rheum 1990;19:269-77.

5. Iyer VN, Joshi AY, Ryu JH. Spontaneous pneumomediastinum: analysis of 62 consecutive adult patients. Mayo Clin Proc 2009;84:417-21.

6. Goff BL, Cherin P, Cantagrel A, Gayraud M, Hachulla E, Laborde $\mathrm{F}$, et al. Pneumomediastinum in interstitial lung disease associated with dermatomyositis and polymyositis. Arthritis Rheum 2009;61:108-18.

7. St. Peter SD, Abbas MA, Kelly KA. The spectrum of pneumatosis intestinalis. Arch Surg 2003;138:68-75.

8. Alexiou K, Sakellaridis T, Sikalias N, Karanikas I, Economou N, Antsaklis G. Subcutaneous emphysema, pneumomediastinum and pneumoperitoneum after unsuccessful ERCP: a case report. Cases J 2009;2:120.

9. Saito M, Tanikawa A, Nakasute K, Tanaka M, Nishikawa T. Additive contribution of multiple factors in the development of pneumatosis intestinalis: a case report and review of the literature. Clin Rheumatol 2007;26:601-3.

J Rheumatol 2010;37:10; doi:10.3899/jrheum.100254 